Vietnam Journal of Mechanics, NCST of Vietnam Vol. 25, 2003, No2 (65-76)

\title{
A SELECTION OF PARAMETERS OF TUNED MASS DAMPER FOR MULTI-DEGREE-OF-FREEDOM SYSTEMS SUBJECTED TO SECOND ORDER COLOURED NOISE EXCITATION
}

\author{
Nguyen Dong Anh ${ }^{1}$ And Nguyen Chi SAng ${ }^{2}$ \\ ${ }^{1}$ Institute of Mechanic Hanoi \\ ${ }^{2}$ Research Institute of Mechanical Engineering
}

\begin{abstract}
Among the fundamental concepts for structure control, passive control systems have a significant role since they do not require additional energies produced by actuator. The basic of passive control is to incorporate a secondary system into the primary structure in order to transfer a part of the vibration energy of the primary system into the secondary one. In the paper the design of an optimal tuned mass damper (TMD) for a structure subjected to second order coloured noise excitation is investigated in order to minimize the sum of response mean squares of components of the primary system with a given ranking priority.
\end{abstract}

\section{Introduction}

Under environmental loading structures and machines may produce large undesired vibrations, which can reflect into their quality and durability. The use of tuned mass dampers (TMD) helps to reduce the undesired vibrations in the primary system.

A single form of TMD for structures and machines was first studied by Frahm (1909) for a single-degree-of-freedom system without damping in both the main and TMD systems. Then the theory of undamped and damped dynamic absorbers in the absence of damping in the main system was studied by Den Hartog (1928). In his study, the basic principle and procedure for proper selection of absorber parameters were developed. The influent of light damping in the main system was investigated by Ioi and Ikeda (1978) by using numerical tools to determine the optimum values of natural frequency as well as damping ratio of TMD. The set of optimum parameters of TMD such as frequency ratio, damping ratio for specified values of the mass ratio and the main system damping ratio was tabulated by Warburton and Ayorinde, see e.g. Soong and Dargush (1997). The investigation for some special vibration systems related to vibration absorbers was investigated by many authors such as Mitropolski, Dao (1994), Muller and Schiehlen (1997), Casciati, Duc, Sang (2002), Papadimitriou and Katafygiotis (1997), Anh and Schiehlen (1994), Roberts and Spanos (1990), Wirsching and Campell (1974), Palazzo et al. (1997). T. Nersessyan, G. Hovhanissian et al. (2001). 
In the paper, the selection of optimum TMD's parameters for vibration systems subjected to second order coloured noise excitation is investigated. The main attention is given to the case of narrow band coloured noise. The well known result for the case of white noise, however, can be obtained as a particular case. The coloured noise is modeled in such a way that covers both narrow band and wide band spectral processes. The main dynamic characteristic of the TMD is selected based on minimizing the sum of response mean squares of components of the primary system.

\section{Second order coloured noise excitation}

The second order coloured noise process $p(t)$ is described as follow

$$
\ddot{p}(t)+2 h \dot{p}(t)+\Omega^{2} p(t)=\sigma \dot{\xi}(t),
$$

where $h, \Omega, \sigma$ are positive constants, $\dot{\xi}(t)$ is the stationary Gaussian white noise process with unit intensity. We investigate a particular case when $\Omega^{2}=\Omega_{1}^{2}+h^{2}$, $\sigma=\sigma_{1} h^{2}$ with $\Omega_{1}, \sigma_{1}$ are positive constants. From equation (2.1) we have

$$
\ddot{p}(t)+2 h \dot{p}(t)+\left(\Omega_{1}^{2}+h^{2}\right) p(t)=\sigma_{1} h^{2} \dot{\xi}(t) .
$$

The spectral density function of $p(t)$ is defined as

$$
S_{p}(\omega)=\frac{\sigma_{1}^{2} h^{4}}{2 \pi\left[4 h^{2}+\left(\Omega_{1}^{2}+h^{2}-\omega^{2}\right)^{2}\right]} .
$$

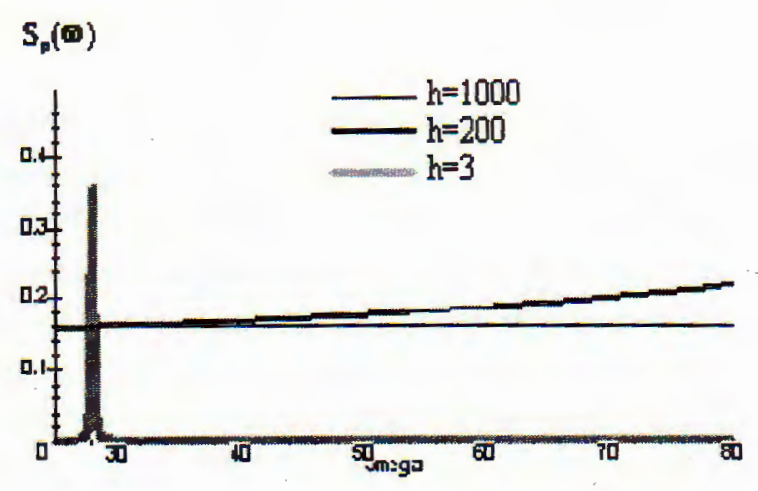

Fig. 1. Plot of $S_{p}(\omega)$ versus frequency $\omega$

It is seen from (2.2) and (2.3) that when $h$ is tending to infinity, $h \rightarrow \infty$, one has:

$$
\begin{aligned}
\lim _{h \rightarrow \infty} p(t) & =\sigma_{1} \dot{\xi}(t), \\
\lim _{h \rightarrow \infty} S_{p}(\omega) & =\frac{\sigma_{1}^{2}}{2 \pi} .
\end{aligned}
$$


Thus, in the limit case, the second order coloured noise $p(t)$ is turned into a white noise process. Hence, the class of second order coloured noise defined by equation (2.2) contains the white noise process as a particular case. Figure 1 illustrates a plot of function $S_{p}(\omega)$ of $p(t)$ with the following parameters $\Omega_{1}=28, \sigma_{1}=1$ and $h=3$, 200,1000 . It is seen that when the parameter $h$ is small enough $p(t)$ is a narrow band process. When $h$ tending to infinity, $h \rightarrow \infty$, the plot getting parallel to the horizontal axe such as a spectral of white noise process. On the other hand, for small values $h$ compared to $\Omega_{1}$ the process $p(t)$ is a narrow band spectral process.

\section{Design of an optimum parameters of TMD}

Figure 2 describes a model for vibration systems subjected to a ground acceleration $-\ddot{y}(t)=p(t)$. This excitation is considered as a second order coloured noise excitation described in the equation (2.1).

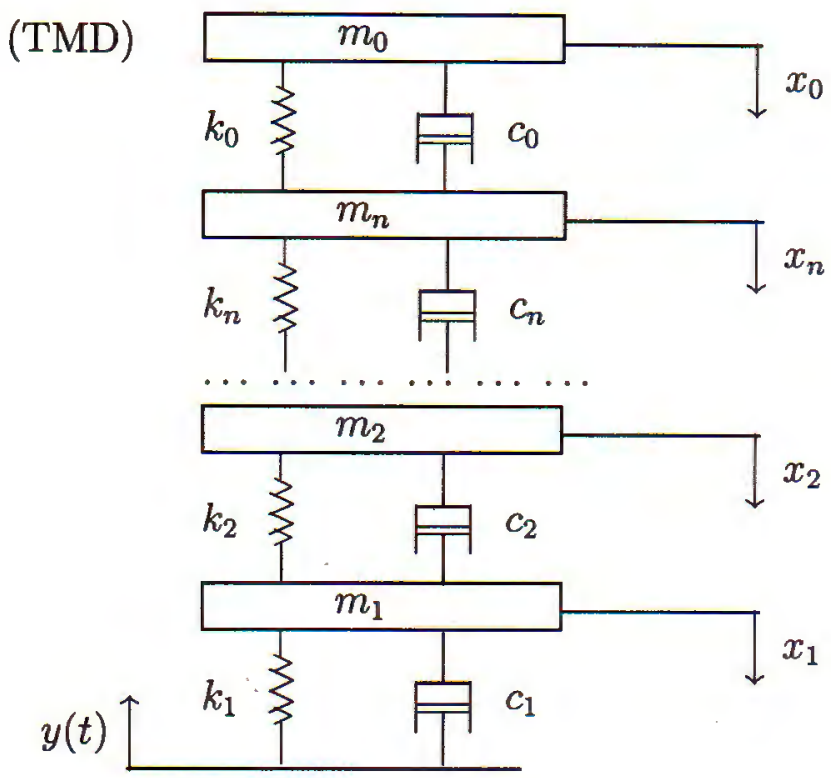

Fig. 2. System under acceleration

The main system consists of $n$ masses $m_{1}, m_{2}, \ldots, m_{n}$ put succeed one to another, each has its stiffness and damping coefficients denoted subsequently as $k_{1}, k_{2}, \ldots, k_{n}$; $c_{1}, c_{2}, \ldots, c_{n}$. The system is subjected to a ground excitation modeled by a second order coloured noise. To suppress undesired large vibrations of the main system, a TMD of mass $m_{0}$ is connected to the mass $m_{n}$ (see Figure 2). Stiffness and damping coefficients of TMD are $k_{0}, c_{0}$.

The equations of motion for the structure with TMD are described by the system of linear differential equations as follows 


$$
M \ddot{X}(t)+C \dot{X}(t)+K X(t)=F(t),
$$

where $M$ is the $(n+1) \times(n+1)$ mass matrix, $X(t)$ is the $(n+1) \times 1$ displacement vector of the structure system relative to its base. $C, K$ are $(n+1) \times(n+1)$ damping and stiffness matrices, respectively

$$
\begin{gathered}
X(t)=\left(x_{1}(t), x_{2}(t), \ldots, x_{n}(t), x_{0}(t)\right)^{T}, \\
M=\left[\begin{array}{ccccc}
m_{1} & 0 & 0 & 0 & 0 \\
0 & m_{2} & 0 & 0 & 0 \\
0 & 0 & \ddots & 0 & 0 \\
0 & 0 & 0 & m_{n} & 0 \\
0 & 0 & 0 & 0 & m_{0}
\end{array}\right], \\
C=\left[\begin{array}{cccccccc}
c_{1}+c_{2} & -c_{2} & 0 & \ldots & 0 & 0 & 0 \\
-c_{2} & c_{2}+c_{3} & -c_{3} & \ldots & 0 & 0 & 0 \\
0 & -c_{3} & c_{3}+c_{4} & \ldots & 0 & 0 & 0 \\
\vdots & \vdots & \vdots & \ddots & \vdots & \vdots & \vdots \\
0 & 0 & 0 & \ldots & c_{n-1}+c_{n} & -c_{n} & 0 \\
0 & 0 & 0 & \ldots & -c_{n} & c_{n}+c_{0} & -c_{0} \\
0 & 0 & & 0 & \ldots & 0 & -c_{0} & c_{0}
\end{array}\right], \\
K=\left[\begin{array}{cccccccc}
k_{1}+k_{2} & -k_{2} & 0 & \ldots & 0 & 0 & 0 \\
-k_{2} & k_{2}+k_{3} & -k_{3} & \ldots & 0 & 0 & 0 \\
0 & -k_{3} & k_{3}+k_{4} & \ldots & 0 & 0 & 0 \\
\vdots & \vdots & \vdots & \ddots & \vdots & \vdots & \vdots \\
0 & 0 & 0 & \ldots & k_{n-1}+k_{n} & -k_{n} & 0 \\
0 & 0 & 0 & \ldots & -k_{n} & k_{n}+k_{0} & -k_{0} \\
0 & 0 & 0 & \ldots & 0 & -k_{0} & k_{0}
\end{array}\right]
\end{gathered}
$$

The base excitation force is

$$
F(t)=\left[\begin{array}{c}
-\ddot{y} m_{1} \\
-\ddot{y} m_{2} \\
\vdots \\
-\ddot{y} m_{n} \\
-\ddot{y} m_{0}
\end{array}\right]
$$


The ground acceleration $(-\ddot{y})$ is considered as the second order coloured noise process $p(t)$ described by the equation (2.1). Introduce notations

$$
\omega_{i}^{2}=\frac{k_{i}}{m_{i}}, \quad \zeta_{i}=\frac{c_{i}}{2 m_{i} \omega_{i}}, \quad \mu_{i}=\frac{m_{i}}{m_{1}}, \quad i=0,1, \ldots, n,
$$

where $\omega_{i}, \zeta_{i}$ are the natural frequency, damping ratio of the separate mass $m_{i}, \mu_{i}$ ratio of mass $m_{i}$ to the mass $m_{1}$.

Transferring equations (3.1) into Ito stochastic differential equations of first order, one can obtain

$$
\begin{aligned}
\dot{X}_{i} & =Y_{i}, \\
\dot{Y}_{i} & =G_{i}(X, Y, p), \\
\dot{p} & =q, \\
\dot{q} & =-2 h q-\Omega^{2} p+\sigma \dot{\xi}(t),
\end{aligned}
$$

where

$$
G(X, Y, q, t)=-M^{-1} C \dot{X}-M^{-1} K X+M^{-1} F(t) .
$$

Using equation system (3.8), one can obtained following moment equations (Roberts and Spanos)

$$
\sum_{i=0}^{n}\left[\left\langle y_{i} \frac{\partial \varphi}{\partial x_{i}}\right\rangle+\left\langle g_{i}(\ldots) \frac{\partial \varphi}{\partial y_{i}}\right\rangle\right]+\left\langle q \frac{\partial \varphi}{\partial p}\right\rangle+\left\langle\left(-2 h q-\Omega^{2} p\right) \frac{\partial \varphi}{\partial q}\right\rangle+\frac{1}{2} \sigma^{2}\left\langle\frac{\partial^{2} \varphi}{\partial q^{2}}\right\rangle=0
$$

where $\langle\ldots\rangle$ is the mathematical expectation. The functions $\varphi$ are selected as follow

$$
\varphi=x_{i} x_{j}, x_{i} y_{j}, x_{i} p, x_{i} q, y_{i} y_{j}, y_{i} q, y_{i} p, q^{2}, p^{2}, p q,
$$

where $i, j=0,1,2, \ldots, n$.

Substituting (3.11) into (3.10), then solving the equation system (3.10), one can get the mean square response of the mass $m_{i}$, as functions off all system parameters

$$
\left\langle x_{i}^{2}\right\rangle=\alpha_{i}\left(\omega_{0}, \omega_{1}, \ldots, \omega_{n}, \zeta_{0}, \zeta_{1}, \ldots, \zeta_{n}, \mu_{0}, 1, \mu_{2}, \ldots, \mu_{n}, h, \sigma, \Omega\right), \quad i=1, \ldots, n .
$$

We denote $\left\langle x_{i 0}^{2}\right\rangle$ as mean square response of the mass $m_{i}$ of the system without TMD. Solving corresponding moment equations one can find

$$
\left\langle x_{i 0}^{2}\right\rangle=\alpha_{i 0}\left(\omega_{1}, \omega_{2}, \ldots, \omega_{n}, \zeta_{1}, \zeta_{2}, \ldots, \zeta_{n}, 1, \mu_{2}, \ldots, \mu_{n}, h, \sigma, \Omega\right), \quad i=1, \ldots, n .
$$


The objective of the study is to select a set of parameters of TMD satisfying the following condition

$$
k=\frac{R}{R_{0}} \Rightarrow \min _{\omega_{0}, \zeta_{0}}
$$

where

$$
\begin{aligned}
& R=\sum_{i=1}^{n} \psi_{i}\left\langle x_{i}^{2}\right\rangle \text { sum of mean squares of response components of the } \\
& \text { main system with TMD, } \\
& R_{0}=\sum_{i=1}^{n} \psi_{i}\left\langle x_{i 0}^{2}\right\rangle \text { sum of mean squares of response components of the } \\
& \quad \text { main system without TMD, }
\end{aligned}
$$

$\psi_{i}$ ranking priorities, $0 \leq \psi_{i} \leq 1, \mu_{0}$ is given.

The ratio $k$ represents the effect of TMD, when $k<1$ the TMD has good effect and when $k>1$ it has bad effect. Substitute (3.12), (3.13), (3.15), (3.16) into (3.14).. Given the values of $\psi_{i}, \omega_{i}, \zeta_{i}, \mu_{i}, \sigma, h, \Omega(i=1,2, \ldots, n)$, the value of $k$ will depend on two parameters $\omega_{0}, \zeta_{0}$. In order to get the smallest value of $k$ we take partial derivatives of $k(\ldots)$ to variables $\omega_{0}, \zeta_{0}$ and set them to zero, one has following equation system

$$
\frac{\partial k\left(\omega_{0}, \zeta_{0}\right)}{\partial \omega_{0}}=0, \quad \frac{\partial k\left(\omega_{0}, \zeta_{0}\right)}{\partial \zeta_{0}}=0 .
$$

Solving equation system (3.18) one finds out a set of optimum parameters: $\omega_{0}, \zeta_{0}$. Substituting the obtained values of $\omega_{0}$ and $\zeta_{0}$ into (3.7), one can get the optimal values of $k_{0}$ and $c_{0}$ of TMD.

\section{Numerical study}

This part of the paper investigates the effects of TMD for two-degree-of-freedom systems subjected to second order coloured noise excitation using numerical method.

Consider a particular vibration structural system described in figure 3 .

It is assumed that the system is subjected to ground acceleration $-\ddot{y}(t)=p(t)$. This excitation is considered as the second order colored noise excitation described in equation (2.1). The equation of motion of the structural system can be written in the form

$$
\begin{aligned}
& {\left[\begin{array}{ccc}
m_{1} & 0 & 0 \\
0 & m_{2} & 0 \\
0 & 0 & m_{0}
\end{array}\right]\left[\begin{array}{l}
\ddot{x}_{1} \\
\ddot{x}_{2} \\
\ddot{x}_{0}
\end{array}\right]+\left[\begin{array}{ccc}
c_{1}+c_{2} & -c_{2} & 0 \\
-c_{2} & c_{2}+c_{0} & -c_{0} \\
0 & -c_{0} & c_{0}
\end{array}\right]\left[\begin{array}{l}
\dot{x}_{1} \\
\dot{x}_{2} \\
\dot{x}_{0}
\end{array}\right]} \\
& +\left[\begin{array}{ccc}
k_{1}+k_{2} & -k_{2} & 0 \\
-k_{2} & k_{2}+k_{0} & -k_{0} \\
0 & -k_{0} & k_{0}
\end{array}\right]\left[\begin{array}{l}
x_{1} \\
x_{2} \\
x_{0}
\end{array}\right]=\left[\begin{array}{l}
m_{1} p(t) \\
m_{2} p(t) \\
m_{0} p(t)
\end{array}\right]
\end{aligned}
$$


Substitute (3.7) into (4.1) and transform equation system (4.1) into Ito stochastic different equations of first order as described in the equation (3.8), where

$$
\begin{aligned}
& g_{1}=\left(-2 \zeta_{1} \omega_{1}-2 \zeta_{2} \omega_{2} \mu_{2}\right) y_{1}+2 \zeta_{2} \omega_{2} \mu_{2} y_{0}-\left(\omega_{1}^{2}+\mu_{2} \omega_{2}^{2}\right) x_{1}+\mu_{2} \omega_{2}^{2} x_{2}+p \\
& g_{2}=-2 \zeta_{2} \omega_{2} y_{1}+2 \zeta_{2} \omega_{2} y_{2}-2 \frac{\mu_{0}}{\mu_{2}} \zeta_{0} \omega_{0}\left(y_{2}-y_{0}+x_{2} \omega_{0}^{2}+x_{0} \omega_{0}^{2}\right)+\omega_{2}^{2} x_{1}-\omega_{2}^{2} x_{2}+p \\
& g_{1}=2 \zeta_{0} \omega_{0}\left(y_{2}-y_{0}\right)-\omega_{0}^{2} x_{0}+p \\
& \dot{p}=q \\
& \dot{q}(t)=-2 h q(t)-\Omega^{2} p(t)+\sigma \dot{\xi}(t) .
\end{aligned}
$$

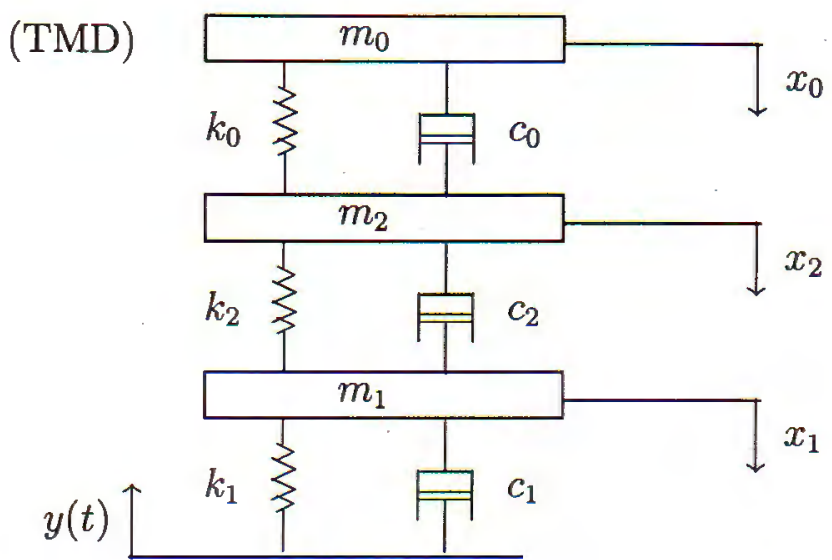

Fig. 3. Model of two-degree-of-freedom system with TMD

Function $\varphi$ indicated in equation (3.11) can be taken as follow:

$$
\begin{aligned}
& \varphi_{1}=x_{1}^{2}, \quad \varphi_{2}=y_{1}^{2}, \quad \varphi_{3}=x_{2}^{2}, \quad \varphi_{4}=y_{2}^{2}, \quad \varphi_{5}=x_{0}^{2}, \quad \varphi_{6}=y_{0}^{2}, \\
& \varphi_{7}=p^{2}, \quad \varphi_{8}=q^{2}, \quad \varphi_{9}=x_{1} x_{2}, \quad \varphi_{10}=x_{1} x_{0}, \quad \varphi_{11}=x_{1} y_{1}, \quad \varphi_{12}=x_{1} y_{2}, \\
& \varphi_{13}=x_{1} y_{0}, \quad \varphi_{14}=x_{1} p, \quad \varphi_{15}=x_{1} q, \quad \varphi_{16}=x_{2} x_{0}, \quad \varphi_{17}=x_{2} y_{1}, \quad \varphi_{18}=x_{2} y_{2}, \\
& \varphi_{19}=x_{2} y_{0}, \quad \varphi_{20}=x_{2} p, \quad \varphi_{21}=x_{2} q, \quad \varphi_{22}=x_{0} y_{1}, \quad \varphi_{23}=x_{0} y_{2}, \quad \varphi_{24}=x_{0} y_{0}, \\
& \varphi_{25}=x_{0} p, \quad \varphi_{26}=x_{0} q, \quad \varphi_{27}=y_{1} y_{2}, \quad \varphi_{28}=y_{1} y_{0}, \quad \varphi_{29}=y_{1} p, \quad \varphi_{30}=y_{1} q, \\
& \varphi_{31}=y_{2} y_{0}, \quad \varphi_{32}=y_{2} p, \quad \varphi_{33}=y_{2} q, \quad \varphi_{34}=y_{0} p, \quad \varphi_{35}=y_{0} q, \quad \varphi_{36}=p q .
\end{aligned}
$$

Substitute (4.2), (4.3) into (3.10), we can obtain 36 equations. The equation system can be solved using MAPLE V.4 to find out unknowns

$$
\left\langle x_{1}^{2}\right\rangle,\left\langle x_{2}^{2}\right\rangle .
$$


In case of structure without TMD, the equations of motion can be described as follow

$$
\begin{aligned}
\left\{\begin{array}{l}
\ddot{x}_{10} \\
\ddot{x}_{20}
\end{array}\right\} & +\left[\begin{array}{cc}
2 \zeta_{1} \omega_{1}+2 \zeta_{2} \omega_{2} \mu & -2 \zeta_{2} \omega_{2} \mu \\
-2 \zeta_{2} \omega_{2} \mu & 2 \zeta_{2} \omega \mu
\end{array}\right]\left\{\begin{array}{l}
\dot{x}_{10} \\
\dot{x}_{20}
\end{array}\right\} \\
& +\left[\begin{array}{cc}
\omega_{1}^{2}+\mu \omega_{2}^{2} & -\mu \omega_{2}^{2} \\
-\omega_{2}^{2} & \omega_{2}^{2}
\end{array}\right]\left\{\begin{array}{l}
x_{10} \\
x_{20}
\end{array}\right\}=\left\{\begin{array}{l}
p(t) \\
p(t)
\end{array}\right\},
\end{aligned}
$$

where $x_{10}, x_{20}$ are displacements of masses $m_{1}, m_{2}$. Using Ito stochastic and moment equations, the values of $\left\langle x_{10}^{2}\right\rangle,\left\langle x_{20}^{2}\right\rangle$ and $R_{0}$ in the equation (3.16) also can be calculated. Following we investigate the system with parameters as $\mu_{2}=0.3, \zeta_{1}=$ $0.25, \omega_{1}=40, \omega_{2}=50, \zeta_{2}=0.1, \Omega=30, \mu_{0}=0.04, h=2, \sigma=1, \psi_{1}=1, \psi_{2}=0$.

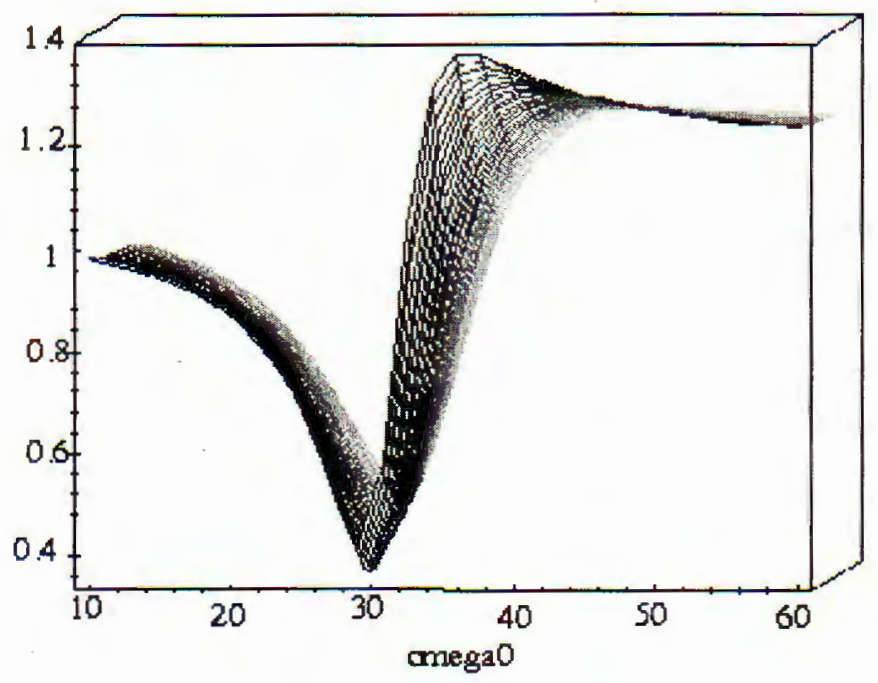

Fig. 4. Plot $3 d$ of $k$ versus $\omega_{0}, \zeta_{0}$

Calculating the optimum parameters of TMD, we find out $k_{\min }=0.35$ at $\omega_{0}=$ $29.25, \zeta_{0}=0.026$. It is clearly that when the primary system is incorporated to TMD with appropriate parameters, the response performance index $k$ is decreased about one third. In case the parameters of TMD are not selected properly, the use of TMD causes the system vibrate more strongly, for example, at $\omega_{0}=36.6 ; \zeta_{0}=0.001$ one has $k=1.38$.

In order to illustrate the above calculated results, the vibration of the system is simulated using software SIMULINK in three cases: without TMD, with optimum TMD and with inappropriate TMD. The simulink diagram is described in Figure 5.

From Figure 6, we can see that with appropriate TMD, the displacement of mass $m_{1}$ is decreased approximately $40 \%$, however when selecting wrong parameters of TMD as seen in Figure 7, the displacement $x_{1}$ is much increased. 


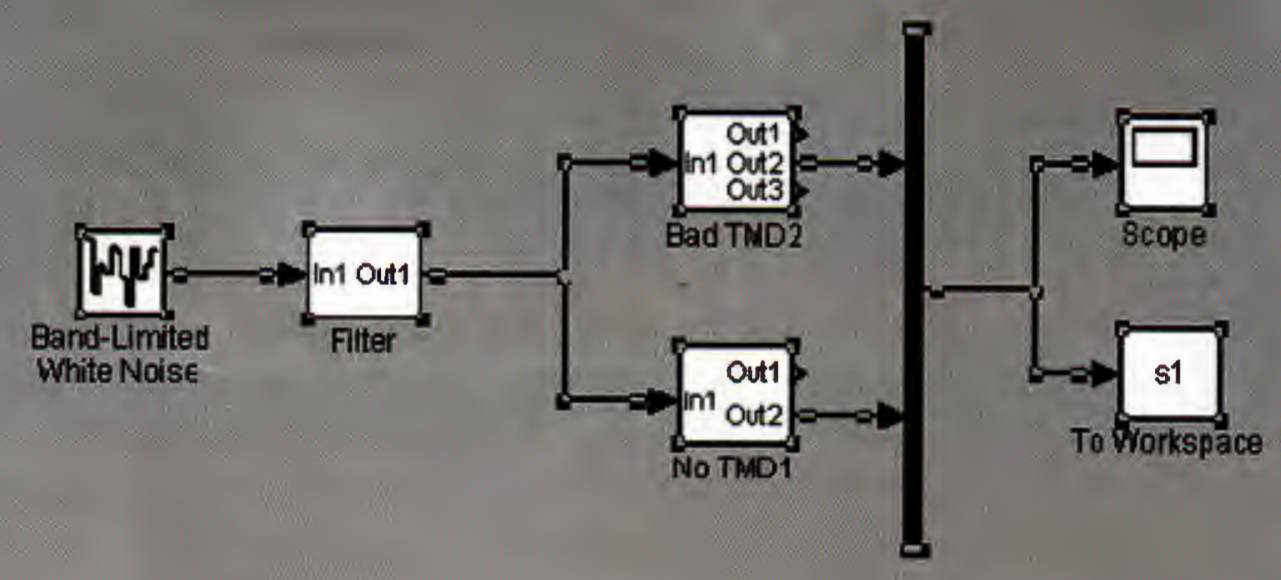

Fig. 5. Model Simulink

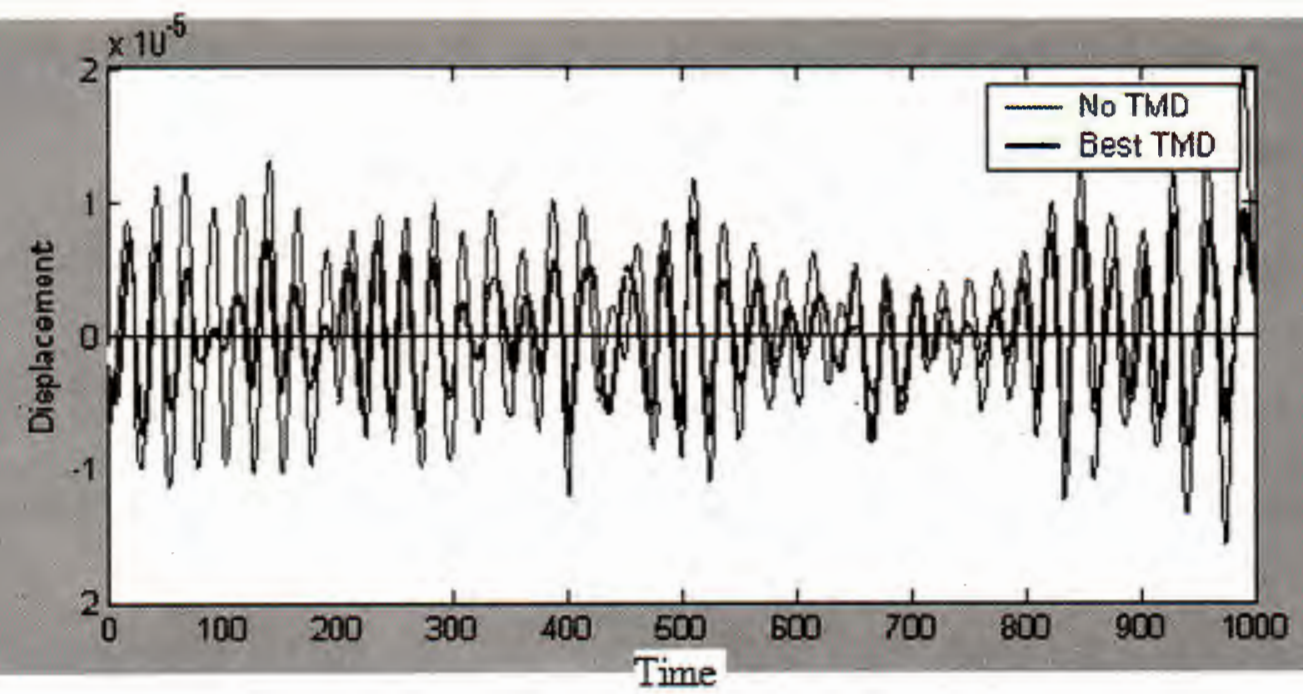

Fig. 6. Displacement of mass $m_{1}$ with optimum and without TMD 


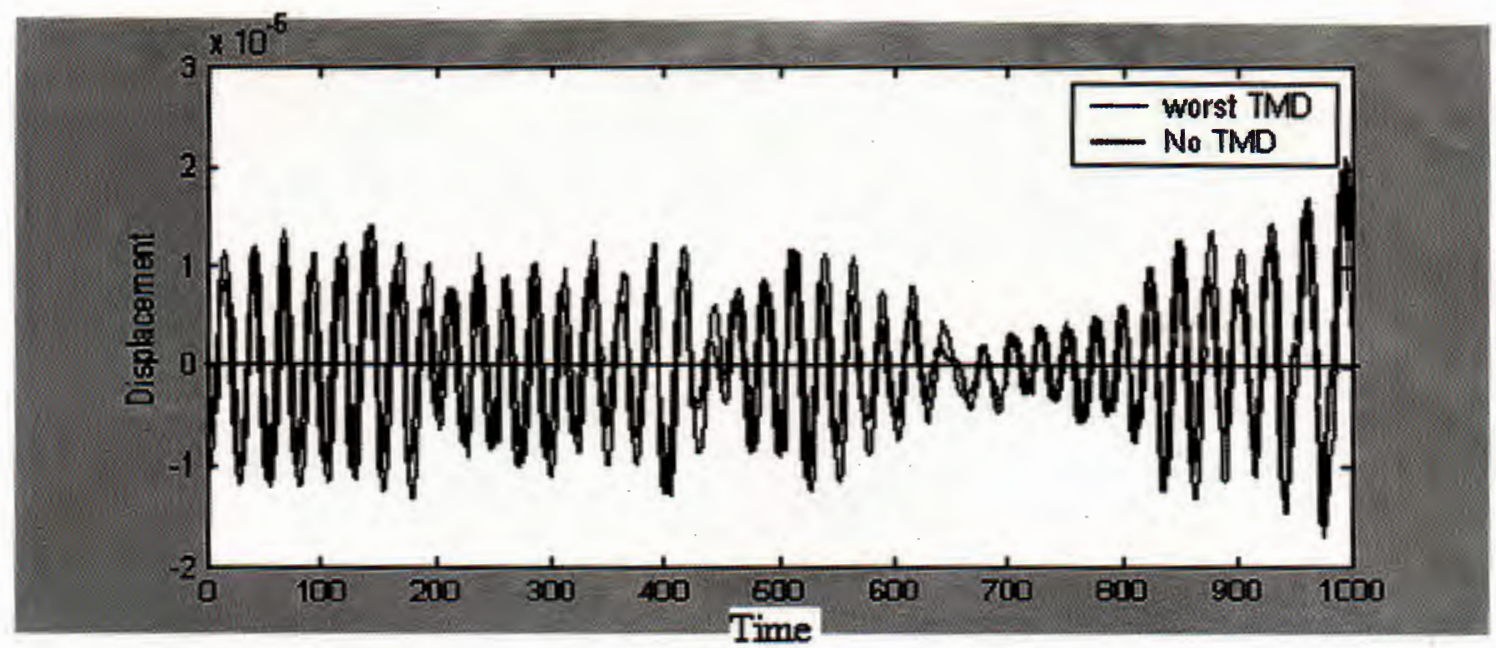

Fig. \%. Displacement of mass $m_{1}$ with worst and without TMD

\section{Particular case}

We investigate a particular case when the system has only mass $m_{1}$ and mass $m_{0}$ is connected to. The damping ratio of the main system is equal to zero $\zeta_{1} \Rightarrow 0$; The structure is subjected to excitation described by equation (2.2)

$$
\ddot{p}(t)+2 h \dot{p}(t)+\left(\Omega_{1}^{2}+h^{2}\right) p(t)=\sigma_{1} \Omega_{1}^{2} \dot{\xi}(t)
$$

and $h \rightarrow \infty$. As studied in part 2, when $h \rightarrow \infty$, the excitation $p(t)$ is turned into white noise process. Solving equation systems (2.4), one can obtain expression of $\left\langle x_{1}^{2}\right\rangle$, then take limitation when $h \rightarrow \infty$, one has:

$$
\begin{aligned}
\lim _{h \rightarrow \infty}\left(\omega_{1}^{3}\left\langle x_{1}^{2}\right\rangle\right)= & \frac{\mu^{4} \lambda^{4}-3 \mu \lambda^{2}-2 \lambda^{2}+4 \zeta_{0}^{2} \lambda^{2}+4 \mu \lambda^{4}+4 \mu^{3} \lambda^{4}+1+12 \zeta_{0}^{2} \mu \lambda^{2}}{4 \lambda \zeta_{2} \mu} \\
& +\frac{12 \zeta_{0}^{2} \mu^{2} \lambda^{2}+4 \zeta_{0}^{2} \mu^{2} \lambda^{2}+\lambda^{4}+6 \mu^{2} \lambda^{4}+\mu^{3} \lambda^{2}}{4 \lambda \zeta_{0} \mu}
\end{aligned}
$$

where $\lambda=\frac{\omega_{0}}{\omega_{1}}$. Taking derivatives (5.1) to variable $\lambda, \zeta_{0}$ and set them to zero, we have equation system:

$$
\begin{aligned}
& \frac{-1+3 \lambda^{4}-2 \lambda^{2}+3 \mu^{4} \lambda^{4}+12 \mu^{3} \lambda^{4}+18 \mu^{2} \lambda^{4}+\mu^{3} \lambda^{2}-3 \mu \lambda^{2}+4 \zeta_{0}^{2} \lambda^{2}+12 \mu \lambda^{4}}{4 \zeta_{2} \lambda^{2} \mu} \\
& +\frac{4 \zeta_{0}^{2} \lambda^{2} \mu^{2}+12 \zeta_{0}^{2} \mu^{2} \lambda^{2}+12 \zeta^{2} \lambda^{2} \mu}{4 \zeta_{0} \lambda^{2} \mu}=0
\end{aligned}
$$




$$
\begin{aligned}
& \frac{4 \zeta_{0}^{2} \lambda^{2} \mu^{3}+12 \zeta_{0}^{2} \lambda^{2} \mu^{2}+12 \zeta_{0}^{2} \lambda^{2} \mu-4 \zeta_{0}^{2} \lambda^{2}+\lambda^{4} \mu^{4}+4 \lambda^{4} \mu^{3}+6 \lambda^{4} \mu^{2}+\lambda^{2} \mu^{3}}{4 \zeta_{0}^{2} \lambda \mu} \\
& +\frac{-3 \lambda^{2} \mu+4 \lambda^{4} \mu-2 \lambda^{2}+1+\lambda^{4}}{4 \zeta_{0}^{2} \lambda \mu}=0 .
\end{aligned}
$$

Solve equation system (5.2), the optimum parameters of TMD can be obtained:

$$
\lambda_{\text {opt }}=\frac{\sqrt{2-\mu}}{\sqrt{2}(1+\mu)}, \quad \zeta_{2 o p t}=\sqrt{\frac{\mu(\mu-4)}{8\left(\mu^{2}-\mu-2\right)}} .
$$

The above result was discovered by Warburton in 1982 when considered structure without damper subjected to white noise excitation.

\section{Conclusions}

There have been many papers investigating the effect of TMD in structural systems for vibration control. This paper presents a procedure for selecting a set of parameters of TMD to minimize sum of mean square of response components with given ranking priority for multi-degree-of-freedom systems.

The numerical study is then investigated for given parameters of primary system and excitation. The result shows that with optimum parameters of TMD, the effectiveness of optimal TMD is very good, however the use of TMD with inappropriate parameters may produce bad effect for the vibration system. This calculation is verified by running simulation using software SIMULINK of MATLAB R12.

This publication is completed with financial support from The Council for Natural Science of Vietnam.

\section{REFERENCES}

1. Anh N. D., Schiehlen W. (1994), An approach to the problem of closure in the non- linear stochastic mechanics. International Journal of Meccanica, 29, 109 123.

2. Casciati F., Duc K. T., Sang N. C. A Selection for Optimal Tuned Mass Dampers, (presented at Third World Conference on Structure Control, Como, Italy, April, 2002).

3. Den Hartog J. P. (1956), Mechanical Vibrations, $4^{\text {th }}$, Edition, McGraw-Hill, NY.

4. Frahm H. (1909), Device for damped vibration of bodies, U. S. Patent No 989958, Oct. 30 .

5. Ioi T., Ideka K. (1978), On the dynamic vibration damped absorber of the vibration system, Bulletin of Japanese Society of Mechanical Engineering, 21 (151), 64-71. 
6. Mitropolski Yu. A., Dao N. V. (1994), Applied asymptotic methods in nonlinear oscillations, Kluwer.

7. Muller P. C. \& Schiehlen W. O. (1997), Linear vibration, Construction Publisher (in Vietnamese).

8. Papapdimitrou C., Katafygiotis L. S. and Siu Kiu Au. (1997), Effect of structural uncertainties on TMD design: a reliability-based approach, Journal of Structural Control, Vol.4, No.1, June.

9. Roberts J. B., Spanos P. D. (1990), Random vibration and stochastic linearization, London, Wiley.

10. Palazzo, B. et al. (1997), Response of base isolated systems equipped with turned mass dampers to random excitations, Journal of Structural Control, vol. 4, N1, 9-22.

11. Soong T. T., Dargush G. F. (1997), Passive energy dissipation systems in structural engineering, John Wiley \& Sons Ltd., West Sussex, England.

12. Nersessyan T., Hovhanissian G., Tonuyan A. and Avanesova K. (2001), Investigations on stiffness - damping interaction for rubber bearings, Journal of Structural Control, Vol. 8, N. 2, pp. 219-235, December.

13. Wirsching P. H., Campell G. W. (1974), Minimal structural response under random excitation using the vibration absorber, Earthquake Engineering and Structural Dynamics. Vol. 2, No. 4, pp. 197-217.

Received November 15, 2002

PHƯƠNG PHÁP LỰA CHỌN CÁC THÔNG SỐ CHO Bộ TMD CHO HỆ NHIỀU BẬC TỰ DO CHỊU KÍCH ĐỘNG ỒN MẦU BẬC HAI

Trong các phương pháp điều khiển hệ kết cấu, phương pháp điều khiển thụ động đóng một vai trò quan trọng vì nó không cần phải dùng ngoại lực. Nguyên lý của phương pháp điều khiển dao động thụ động là gắn vào hệ kết cấu chính một hệ thứ cấp để phân tán một phần năng lượng dao động từ hệ chính sang hệ thứ cấp. Bài báo đề cập đến một phương pháp tính toán tối ưu bộ TMD cho hệ kết cấu nhiều bậc tự do chịu kích động ồn mầu bậc hai nhằm đạt được giá trị nhỏ nhất của tổng các đáp ứng bình phương trung bình với thứ tự ưu tiên cho trước. 